\title{
Smooth muscle phenotypic modulation is an early event in aortic aneurysms
}

\author{
Gorav Ailawadi, MD, ${ }^{\text {a }}$ Christopher W. Moehle, BS, ${ }^{\mathrm{b}}$ Hong Pei, MD, ${ }^{\text {a }}$ Sandra P. Walton, BS, ${ }^{\text {a }}$ \\ Zequan Yang, MD, PhD, ${ }^{a}$ Irving L. Kron, MD, ${ }^{a}$ Christine L. Lau, MD, ${ }^{a}$ and Gary K. Owens, $\mathrm{PhD}^{\mathrm{b}}$
}

Objectives: Vascular smooth muscle cells can undergo profound changes in phenotype, defined by coordinated repression of smooth muscle cell marker genes and production of matrix metalloproteinases in response to injury. However, little is known of the role of smooth muscle cells in aortic aneurysms. We hypothesized that smooth muscle cells undergo phenotypic modulation early in the development of aortic aneurysms.

\begin{abstract}
Methods: Abdominal aortas from C57B6 mice $(\mathrm{n}=79)$ were perfused with elastase or saline (control) and harvested at $1,3,7$, or 14 days. Aortas were analyzed by means of quantitative polymerase chain reaction and immunohistochemistry for smooth muscle cell marker genes, including SM22A, smooth muscle $\alpha$-actin, and matrix metalloproteinases 2 and 9. In complimentary experiments human aneurysms $(n=10)$ and control aorta $(n=10)$ were harvested at the time of surgical intervention and analyzed.

Results: By 14 days, aortic diameter was larger after elastase perfusion compared with control diameter $(100 \% \pm$ $9.6 \%$ vs $59.5 \% \pm 18.9 \%, P=.0002$ ). At 7 days, elastase-perfused mice had a $78 \%$ and $85 \%$ reduction in SM22 $\alpha$ and smooth muscle $\alpha$-actin expression, respectively, compared with that seen in control animals well before aneurysms were present, and these values remained repressed at 14 days. Immunohistochemistry confirmed less SM22 $\alpha$ and smooth muscle $\alpha$-actin in experimental aneurysms at 14 days in concert with increased matrix metalloproteinase 2 and 9 expression at 7 and 14 days. Similarly, human aneurysms had less SM22 $\alpha$ and smooth muscle $\alpha$-actin and increased matrix metalloproteinase 2 and 9 staining, compared with control values, as determined by means of quantitative polymerase chain reaction.
\end{abstract}

Conclusions: Aneurysms demonstrate smooth muscle cell phenotypic modulation characterized by downregulation of smooth muscle cell marker genes and upregulation of matrix metalloproteinases. These events in experimental models occur before aneurysm formation. Targeting smooth muscle cells to a reparative phenotype might provide a novel therapy in the treatment of aortic aneurysms. ( $\mathrm{J}$ Thorac Cardiovasc Surg 2009;138:1392-9)

The mechanisms involved in aortic aneurysm formation are not well understood. An unknown inciting event results in aortic wall injury, whereby leukocytes are recruited into the aortic wall. ${ }^{1}$ In response to local inflammatory cytokine production, macrophages and smooth muscle cells (SMCs) release proteases, including matrix metalloproteinases (MMPs), leading to destruction of the extracellular matrix proteins collagen and elastin. ${ }^{1}$ Late in the course of aneurysms, SMCs undergo apoptosis, thereby losing the cells primarily responsible for the synthesis of extracellular matrix proteins. The ongoing shift toward degradation of collagen

From the Departments of Surgery ${ }^{\mathrm{a}}$ and Molecular Physiology, ${ }^{\mathrm{b}}$ University of Virginia, Charlottesville, Va.

Read at the Eighty-ninth Annual Meeting of The American Association for Thoracic Surgery, Boston, Mass, May 9-13, 2009.

Supported by TSFRE research grant 2008-2010 (to G.A.).

Received for publication May 20, 2009; revisions received June 30, 2009; accepted for publication July 29, 2009.

Address for reprints: Gorav Ailawadi, MD, TCV Surgery, PO Box 800679, Charlottesville, VA 22908 (E-mail: gorav@virginia.edu).

$0022-5223 / \$ 36.00$

Copyright (c) 2009 by The American Association for Thoracic Surgery doi:10.1016/j.jtcvs.2009.07.075 and elastin is thought to be an important concept in the progression of aneurysms. ${ }^{1}$

It is well established that vascular SMCs have the ability to undergo profound changes in phenotype in response to changes in their extracellular environment, as occurs after vascular injury and in atherosclerosis in both preclinical animal models and human subjects. ${ }^{2}$ These changes often include enhanced proliferation and migration, as well as marked changes in gene expression patterns, including coordinated repression of SMC marker genes and induction of MMPs in response to inflammatory mediators. ${ }^{2,3}$ Specifically, repression of SM22 $\alpha$, smooth muscle (SM) $\alpha$-actin, and smooth muscle myosin heavy chain in concert with increased MMP-2, MMP-3, and MMP-9 expression is seen in patients with atherosclerosis. ${ }^{2}$ These and other alterations in SMC phenotype are thought to play a key role in the pathophysiology of multiple disorders, including atherosclerosis, restenosis, tumor metastasis, and hypertension. ${ }^{2,3}$ The focus of much of the work related to the development of aortic aneurysms has been on the role of macrophages and leukocytes, whereas little is known of the role of SMCs. 

Abbreviations and Acronyms
$\mathrm{CABG}=$ coronary artery bypass grafting
$\mathrm{KLF}=$ Krüppel-like factor
MMP = matrix metalloproteinase
$\mathrm{qPCR}=$ quantitative polymerase chain reaction
$\mathrm{SM}=$ smooth muscle
SMC $=$ smooth muscle cell
$\mathrm{TGF}=$ transforming growth factor

We hypothesized that SMCs undergo phenotypic switching early during aneurysm formation and contribute to the destruction of aortic wall matrix in response to local inflammatory mediators. Our overall hypothesis is that SMCs, by altering the phenotype to a differentiated or reparative state, might be a target to treat aortic aneurysms.

\section{MATERIALS AND METHODS Murine Aneurysm Model}

The elastase perfusion model to create abdominal aortic aneurysms in mice has been well described. ${ }^{4-6}$ In this well-accepted model aortas dilate by $30 \%$ to $50 \%$ initially after perfusion with elastase or saline. Elastase perfusion results in progressive aortic dilation such that an aneurysm of $100 \%$ over baseline develops by 14 days (Figure $1, B) .^{4-6}$ In contrast, saline-perfused aortas do not dilate following perfusion. Briefly, the infrarenal abdominal aorta is isolated in situ. Branches are ligated with 7-0 monofilament sutures. After occluding the aorta proximally and distally, an arteriotomy is made at the aortic bifurcation with a 31-gauge needle. The aorta is cannulated and perfused with porcine pancreatic elastase $(0.47 \mathrm{U} /$ $\mathrm{mL}$ ) for 5 minutes at a pressure of $100 \mathrm{~mm} \mathrm{Hg}$ (Figure 1, A). Control animals are perfused with normal saline $(0.9 \%)$ for 5 minutes. The arteriotomy is repaired with interrupted $10-0$ monofilament sutures, and flow is reestablished in the aorta. The abdominal contents are replaced, the abdominal wall is closed, and the mice are recovered. Video micrometric measurements of the aortic wall diameter are made in situ before perfusion, after perfusion, and at harvest. All animal protocols described were approved by the University of Virginia Animal Care and Use Committee (protocol no. 3634).

The aortas from wild-type C57B6 mice $(n=79)$ were perfused with elastase or saline (control). Saline-treated aortas were harvested at 1 $(n=9), 3(n=9), 7(n=8)$, or $14(n=10)$ days. Similarly, elastase-treated aortas were harvested at $1(n=9), 3(n=10), 7(n=12)$, or $14(n=12)$ days. Aortas were analyzed by means of real-time quantitative polymerase chain reaction (qPCR) or immunohistochemistry.

\section{qPCR}

Aortas were harvested, flash-frozen in liquid nitrogen, and held at $-80^{\circ} \mathrm{C}$ until extraction. RNA was extracted with Trizol (Invitrogen, Carlsbad, Calif). Briefly, for mice, RNA was extracted from frozen tissue by pulverizing in Trizol with the Pellet Pestle handheld homogenizer (Thermo Fisher Scientific, Waltham, Mass). Human tissue was crushed under liquid nitrogen with a mortar and pestle and further homogenized in Trizol by using a bead mill (Fast Prep 24; MP Biomedicals, Solon, Ohio). cDNA was synthesized from extracted RNA with the QuantiTect Reverse Transcription Kit (Qiagen, Inc, Valencia, Calif). qPCR was performed with TaqMan Gene Expression probe sets in conjunction with TaqMan Gene Expression Master Mix (Applied Biosystems, Inc, Foster City, Calif). Primers and probes used were as follows: 18s (both mouse and human) forward, CGGCTACCACATCCAAGGAA; 18s reverse, AG
CTGGAATTACCGCGGC; FAM-labeled probe, TGCTGGCACCA GACTTGCCCTC. Probe sets were for murine SM22A (reference no. Mm00441660-m1), murine SM $\alpha$-actin (reference no. Mm01546133-m1), and murine MMP-2 (reference no. Mm00439506-m1). Human SM $\alpha$-actin (reference no. Hs00909449_m1) and human MMP-2 (reference no. Hs00234422_m1) were purchased from Applied Biosystems. Gene expression was calculated by using the relative quantification method according to the following equation: $2^{(-\Delta \mathrm{CT})}$, where $\Delta \mathrm{CT}=$ (Average gene of interest) - (Average reference gene), where ribosomal $18 \mathrm{~s}$ was used as the reference gene.

\section{Immunohistochemistry}

For immunohistochemistry, murine and human aortas were cut into $5 \mu \mathrm{m}$ paraffin sections. Antibodies for immunohistochemistry were as follows: MMP-2 (R\&D Systems, Inc, Minneapolis, Minn), MMP-9 (R\&D Systems), SM22 $\alpha$ (ABCAM, Inc, Cambridge Mass), and SM $\alpha$-actin (Sigma Cy3-labeled clone 1A4, Product number C6198). To assess for SMC apoptosis and proliferation, caspase-3 (Cell Signaling, Danvers, Mass) and Ki-67 (Santa Cruz, Santa Cruz, Calif) staining was performed. In brief, after antigen retrieval (Vector Laboratories, Burlingame, Calif) with heat-induced epitope retrieval, the primary antibodies were detected with the Vectastain Elite Kit (Vector Laboratories). Visualization was done with DAB (DAKO North America, Inc, Carpinteria, Calif). For murine SM $\alpha$-actin, DAPI (Vectashield HardSet Mountin Medium with DAPI, catalog no. H-1500) counterstaining was used (Vector Laboratories). For human SM $\alpha$-actin, Permanent Red Tables and Substrate Buffer (DAKO) were used. Counterstaining was done with Harris Hematoxylin 1 (Richard-Allen Scientific, Kalamazoo, Mich). For caspase-3, Methyl Green (DAKO) counterstaining was used. Negative controls were run with the omission of the primary antibody. Images were acquired by using the $4 \times, 10 \times$, and $25 \times$ objectives on a Zeiss microscope (Zeiss, Peabody, Mass) equipped with an AxioCam digital camera (Zeiss) with the AxioCam version 4.6 software program in the University of Virginia Cardiovascular Research Center.

For histochemistry, $5 \mu \mathrm{m}$ paraffin sections were assessed for histopathology by using a modified Russell-Movat pentachrome method and hematoxylin and eosin (Laboratory Methods in Histotechnology, Armed Forces Institute of Pathology). Movat- and hematoxylin and eosin-stained slides were imaged as above at the Academic Computing Health Sciences Center at the University of Virginia.

\section{Human Tissue Harvest}

The collection of human aortic aneurysm tissue was approved by the University of Virginia institutional review board (no. 13178). Patients undergoing open surgical operations for ascending or abdominal aortic aneurysm repair or coronary artery bypass grafting $(\mathrm{CABG})$ were consented. The ascending or abdominal aortic aneurysmal wall $(\mathrm{n}=10$ each) was resected at the time of the operation and immediately snap-frozen and later analyzed by means of qPCR and immunohistochemistry. Control tissue was only obtained from the ascending aorta during $\mathrm{CABG}$ procedures $(\mathrm{n}=10)$ from the proximal aortotomy. Patients who had evidence of atherosclerotic ascending aorta, ascending aortic aneurysm, or any known history of collagen vascular disease or aneurysm were excluded from collection as control subjects.

\section{Statistical Analysis}

Comparisons were made between elastase- and saline (control)-perfused animals. Similarly, human aneurysms and control aortas were compared. Statistical analysis was performed with an unpaired, 2-tailed, Mann-Whitney test by using GraphPad Prism 5 software (GraphPad Software, La Jolla, Calif). Data are represented as the mean ( \pm standard error of the mean) of the subject group versus the mean ( \pm standard error of the mean) of the control group. Data were subjected to the Grubb's test to detect any outliers (GraphPad Software). 

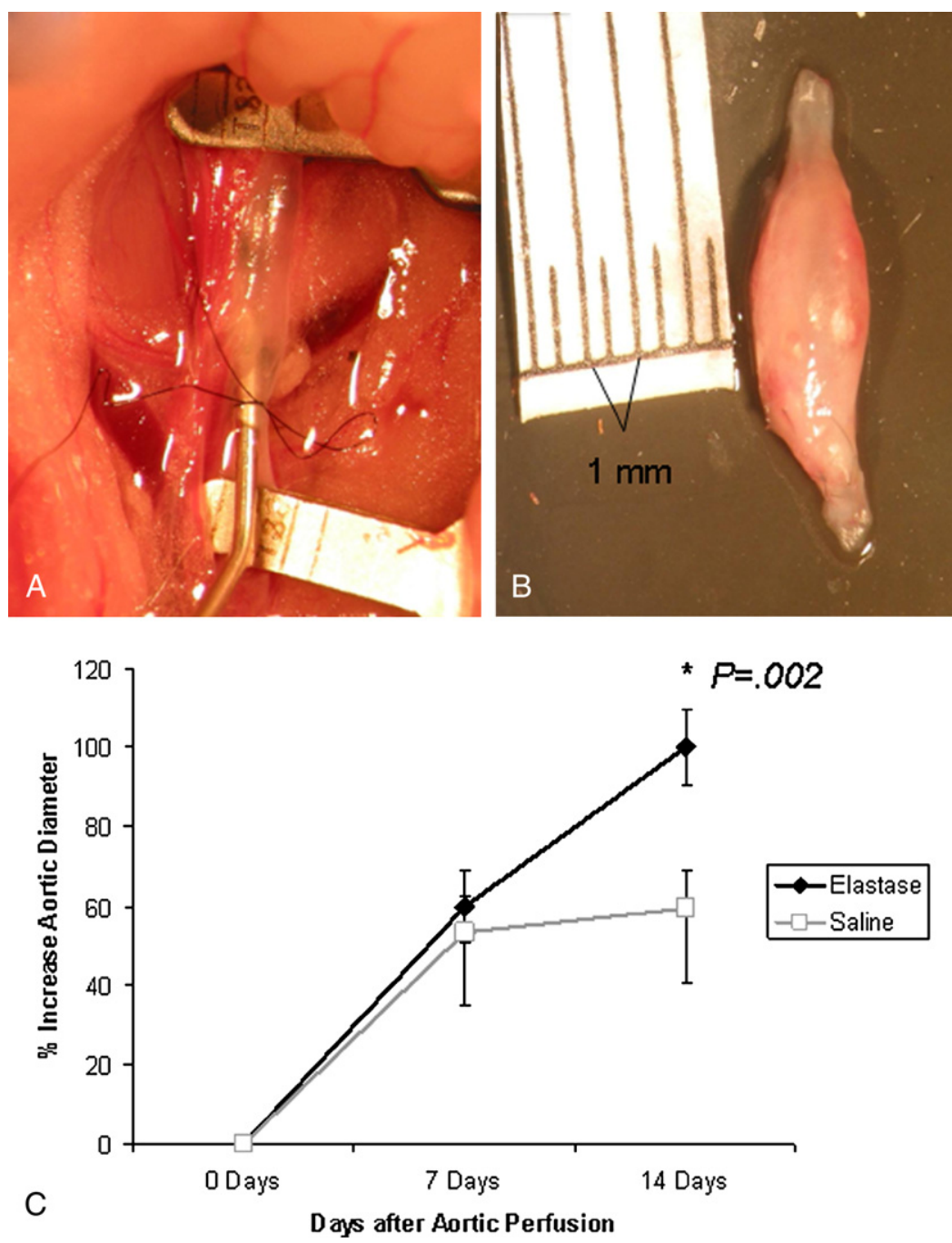

FIGURE 1. A, Elastase perfusion of the infrarenal abdominal aorta. B, An explanted abdominal aortic aneurysm 14 days after elastase perfusion. C, Graph demonstrating aortic aneurysm formation at 14 days after elastase (and not saline) perfusion. Importantly, at 7 days, no differences in aortic diameter are evident between elastase- and saline-treated animals.

\section{RESULTS}

\section{Aortic Aneurysms Develop at 14 Days}

The baseline aortic diameters in mice before and immediately after elastase perfusion were equivalent to the aortic diameters in saline-perfused mice. At 7 days after surgical intervention, the aortic diameter in elastase-perfused mice remained similar to that seen in saline-perfused mice $(60.0 \% \pm 9.13 \%$ vs $53.3 \% \pm 18.3 \%, P=.49$; Figure 1 , $C)$. However, by 14 days, the aortic diameter was significantly larger after elastase perfusion $(100 \% \pm 9.6 \%$ vs $59.5 \% \pm 18.9 \%, P=.0002$; Figure $1, C)$.

\section{Decreased SM Gene Expression Occurs Before Experimental Aneurysms}

qPCR was used to determine the gene expression profiles of aortas after elastase or saline perfusion. At 1 and 3 days after surgical intervention, no differences in expression of the SM marker genes SM $\alpha$-actin or SM22A were identified (data not shown). At 7 days, however, phenotypic modulation of SMCs was evident because elastase-perfused mice had a $78 \%$ reduction in SM22 $\alpha$ expression $(3.22 \mathrm{E}-05 \pm$ $9.18 \mathrm{E}-06$ vs $1.49 \mathrm{E}-04 \pm 2.4 \mathrm{E}-05, P=.003)$ compared with that seen in saline-perfused animals, well before the aneurysm phenotype was present (Figure 2). In addition, $\mathrm{SM} \alpha$-actin expression was $85 \%$ less in aortas from elastasetreated animals compared with that seen in saline-treated animals $(7.19 \mathrm{E}-05 \pm 1.88 \mathrm{E}-05$ vs $4.65 \mathrm{E}-04 \pm 1.41 \mathrm{E}-04$, $P=.034$, Figure 2). MMP-2 expression was increased by $80 \%$ in elastase-treated animals compared with that seen in saline control-treated animals $(1.24 \mathrm{E}-04 \pm 4.02 \mathrm{E}-05$ vs $6.89 \mathrm{E}-05 \pm 1.06 \mathrm{E}-05, P=.044$, data not shown).

At 14 days, SM22 $\alpha$ (3.19E-05 $\pm 8.10 \mathrm{E}-06$ vs $3.31 \mathrm{E}-04 \pm$ $8.85 \mathrm{E}-05, P=.004)$ and $\mathrm{SM} \alpha$-actin $(2.35 \mathrm{E}-04 \pm 4.69 \mathrm{E}-05$ 

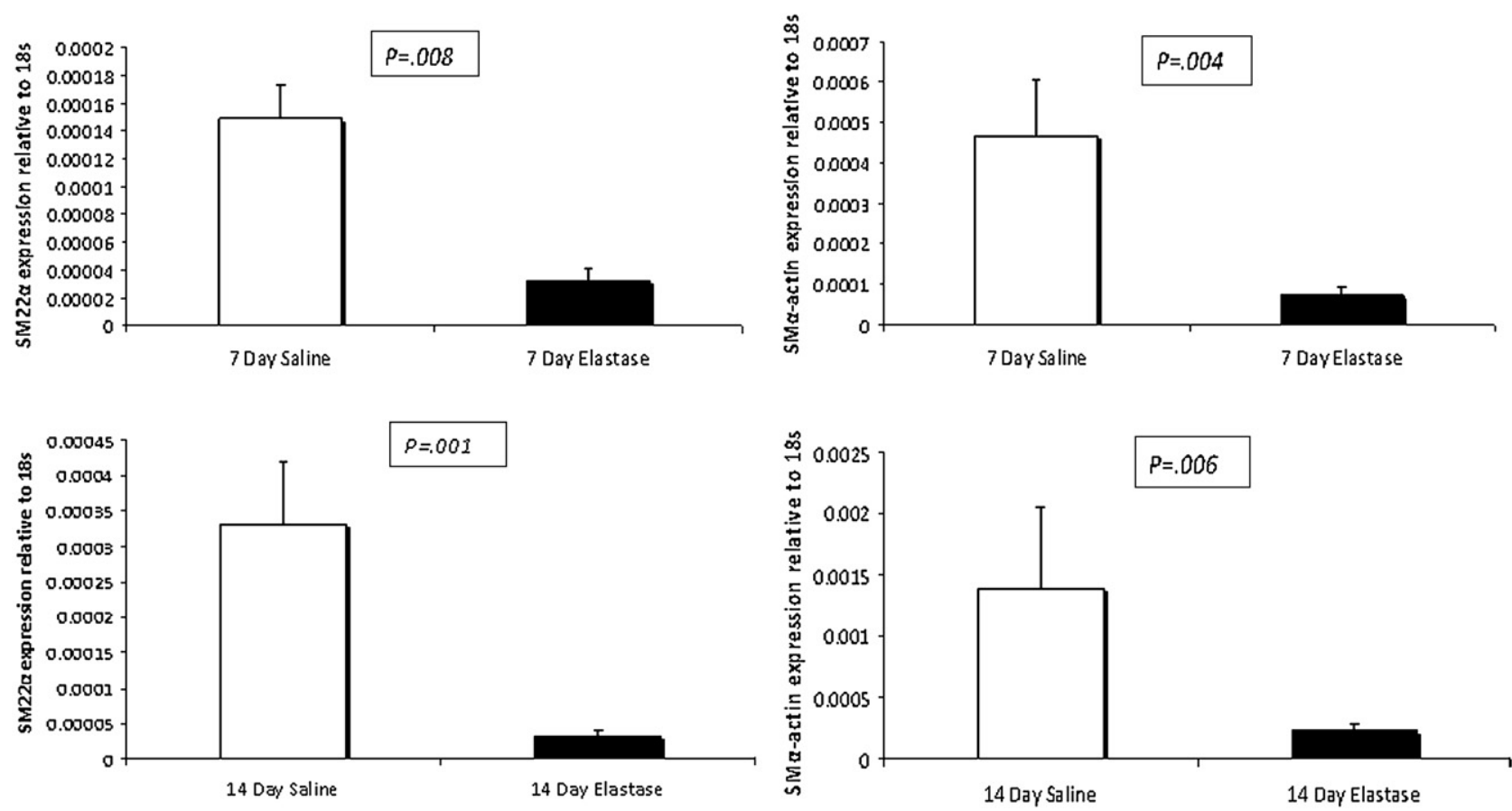

FIGURE 2. Quantitative polymerase chain reaction analysis of smooth muscle marker genes at 7 and 14 days after elastase/saline perfusion. SM $22 \alpha$ and SM $\alpha$-actin expression is downregulated in elastase-treated aortas at 7 and 14 days after perfusion. Standard error bars are shown.

vs $1.39 \mathrm{E}-03 \pm 6.67 \mathrm{E}-04, P=.023$ ) expression remained $90 \%$ and $83 \%$ less, respectively, in aneurysmal aortas compared with that seen in saline-treated aortas (Figure 2). In addition, MMP- 2 expression was $240 \%$ higher 14 days after elastase perfusion compared with that seen after saline perfusion (1.56E-04 $\pm 3.52 \mathrm{E}-05$ vs $4.59 \mathrm{E}-05 \pm 1.77 \mathrm{E}-05$, $P=.026$, data not shown).

Immunohistochemistry did not demonstrate discernable differences in SM22 $\alpha$ or SM $\alpha$-actin expression at 1 or 3 days (data not shown). In addition, minimal differences in the expression of these SM marker genes were evident at 7 days by means of histology. Evidence for some downregulation of SM22 $\alpha$ was seen in some sections but was not marked (blue arrowheads, Figure 3). However, at 14 days, immunohistochemistry did confirm markedly less SM $22 \alpha$ and SM $\alpha$-actin in the media of experimental aneurysms compared with that seen in saline-treated mice (Figure 4). MMP-2 and MMP-9 levels were increased in the media and adventitia at 7 and 14 days but not at 1 or 3 days in elastase-treated aortas compared with those seen in salinetreated aortas (Figures 3 and 4). Interestingly, careful examination of immunohistochemistry demonstrated that, consistent with repression of SMC markers being an integral characteristic of aortic dilation and remodeling, some decrease in SMC marker gene expressions is evident in saline-perfused animals compared with that seen in a normal noninjured aorta. However, this reduction in SMC marker gene expression was consistently and noticeably less than that seen in elastase-perfused animals at 14 days. Impor- tantly, apoptosis, as assessed by cleaved (active) caspase-3 staining at 7 days, was minimal in both elastase- and saline-perfused aortas, suggesting that the observed decrease in SMC marker gene expression was not due to active cell loss (Figure 3). These images were analyzed with Image Pro Plus 4.5 software for the percentage of the vessel wall positive for caspase-3. Positive staining was not increased in elastase-perfused mice $(0.04 \% \pm 0.01 \%)$ compared with that seen in saline-perfused mice $(0.5 \% \pm 0.2 \%)$. Similarly, there was little evidence of apoptosis at 14 days.

\section{Decreased SM Gene Expression in Ascending and Abdominal Aneurysms in Human Subjects}

Human ascending aortic aneurysms harvested at the time of open surgical resection had 98\% less SM $\alpha$-actin expression compared with that seen in control ascending aortic tissue obtained during CABG (aneurysm, 1.48E-06 \pm 6.33E-07; control, 9.08E-05 \pm 5.86E-05; $P=.0026$; Figure 4). In addition, MMP-2 expression was increased by more than $1600 \%$ in aneurysms compared with that seen in control tissue $(6.33 \mathrm{E}-04 \pm 3.17 \mathrm{E}-04$ vs $3.60 \mathrm{E}-05$ $\pm 9.83 \mathrm{E}-06, P=.0003$, Figure 5$)$. As determined by means of immunohistochemistry, SM22 $\alpha$ and SM $\alpha$-actin staining were markedly decreased in the aortic wall from both ascending and abdominal aneurysms compared with that seen in control aortas (Figure 6). In addition, immunohistochemistry demonstrated more medial and adventitial MMP-2 and MMP-9 in human aneurysms compared with that seen in control aortas (Figure 6). Evidence for apoptosis 

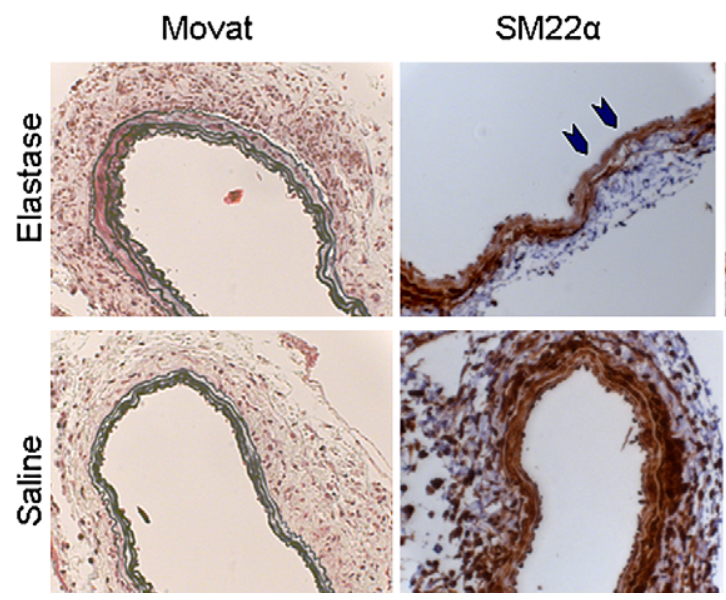

MMP-2
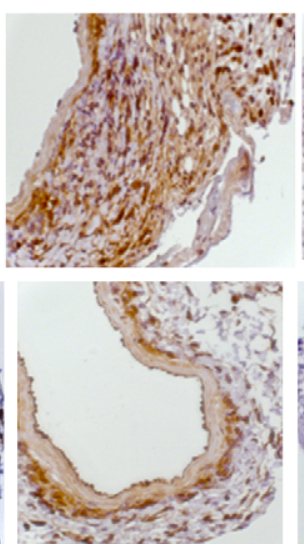

MMP-9
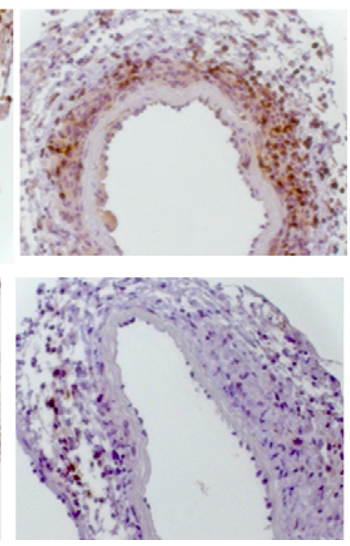

Caspase-3
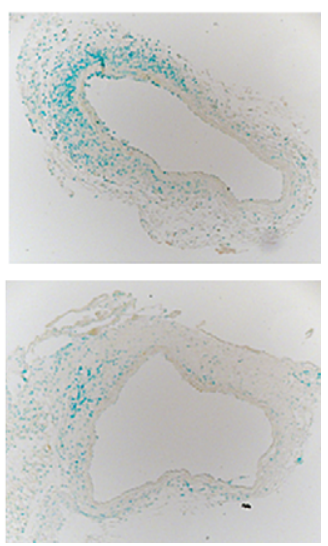

FIGURE 3. Histochemistry and immunohistochemistry in mice 7 days after elastase or saline perfusion. Little differences in elastin are evident by means of Movat staining, with intact elastin in both treatment groups. Some early degradation might be present in regions of the aorta (blue arrowheads) after elastase perfusion compared with that seen after saline perfusion. Expression of matrix metalloproteinase (MMP) 2 and 9 appears to be upregulated at 7 days after elastase compared with that seen after saline. Minimal activated (cleaved) caspase-3 staining is present in either group (brown positive stain, methyl green nuclear counterstain). Images were obtained at $25 \times$ magnification, except for caspase- 3 , which was obtained at $10 \times$ magnification.

obtained by means of Caspase- 3 staining was present in aneurysmal aortas, with less evidence for apoptosis in control aortic tissue. It is likely in these advanced aneurysms requiring resection that cellular loss can explain at least some of the decreased SMC marker gene expression.

\section{DISCUSSION}

The role of SMCs during the development and progression of aortic aneurysms is not well defined. In this study we demonstrate a decrease in SM marker genes in advanced human ascending and abdominal aneurysms compared with control tissue from the ascending aorta. These findings might be explained by SMC apoptosis. To test the hypothesis that SMC phenotypic switching occurs early in aneurysm formation, we used a murine model and demonstrated that
SMCs undergo phenotypic modulation early before aneurysm formation, and this does not appear to be explained by the loss of SMCs caused by apoptosis. To prove that SMC phenotypic modulation occurs during progression of aneurysmal disease in human subjects would require access to small aneurysmal aortic tissue in which SMCs are still prevalent or access to a specific marker for phenotypically modulated SMCs, which is yet to be reported. In advanced aneurysms, such as those treated with surgical repair, many SMCs have undergone apoptosis. ${ }^{1}$

Vascular SMCs in adults are plastic and can undergo reversible changes in their phenotype in response to changing environmental cues. ${ }^{2}$ In response to vascular injury, the SMC increases its rate of cell proliferation and migration and its ability to synthesize extracellular matrix, playing
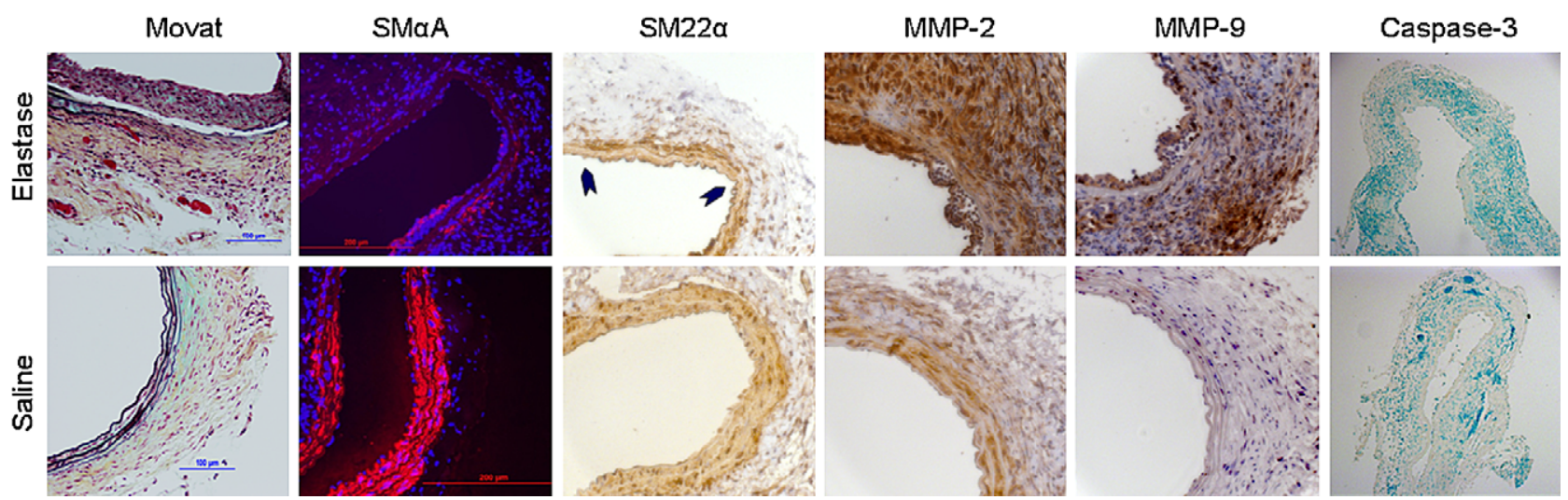

FIGURE 4. Histochemistry and immunohistochemistry in mice 14 days after elastase or saline perfusion. Movat staining demonstrated destruction of the medial elastin layer in elastase-treated animals. SM $\alpha$-actin and SM22 $\alpha$ (arrowheads) are downregulated compared with that seen in saline controls. Expression of matrix metalloproteinase (MMP) 2 and 9 is markedly increased in elastase-treated animals. There is little activated (cleaved) caspase-3 staining in either elastase- or saline-treated animals (brown staining positive, methyl green nuclear counterstain). Images were obtained at $25 \times$ magnification, except for caspase-3, which was obtained at $10 \times$ magnification. 

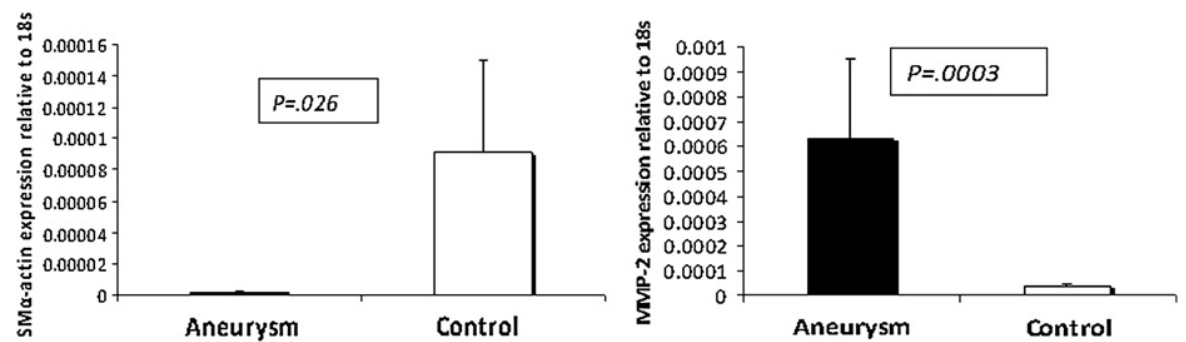

FIGURE 5. Quantitative polymerase chain reaction in human ascending aortic aneurysms and control ascending aortic tissue. SM $\alpha$-actin expression is significantly decreased in ascending aortic aneurysms, whereas matrix metalloproteinase $(M M P) 2$ expression is increased compared with that seen in control aortas. Standard error bars are shown.

a critical role in vascular repair. ${ }^{2}$ Teleologically, the ability of SMCs to remain plastic likely conferred a survival advantage for repair of injured vessels. ${ }^{2,3}$ However, the negative effect of this plasticity is that it can predispose the SMC to potentially detrimental phenotypic modulation in response to abnormal environmental cues and can contribute to the progression of vascular disease. ${ }^{2,3}$

This concept of phenotypic modulation has been well studied in models of atherosclerosis and restenosis. Balloon injury of the murine and rabbit carotid artery results in phenotypic switching of SMCs characterized by loss of SM marker genes, including SM22A, SM $\alpha$-actin, and SM myosin heavy chain, concurrent with proliferation and migration of SMCs to the lesion. ${ }^{7}$ These findings have also been observed in human lesions at autopsy after balloon angioplasty. ${ }^{8}$
The exact mechanisms involved in regulation of SM phenotype remain to be elucidated. In vitro and in vivo models have shown that expression of these SM marker genes is dependent on multiple $\mathrm{CC}(\mathrm{A} / \mathrm{T}) 6 \mathrm{CG}$ elements and their binding factor, serum response factor. ${ }^{9-11}$ The serum response factor and $\mathrm{CC}(\mathrm{A} / \mathrm{T}) 6 \mathrm{CG}$ axis appears to be required for SMC phenotypic modulation in vascular injury., ${ }^{3,12}$ Evidence from our laboratory also has shown that a G/C repressor element within the SM22A promoter mediates transcriptional repression of this gene in experimental atherosclerosis. ${ }^{3,13}$ A number of transcriptional pathways, including Krüppel-like factor (KLF) 4, have been shown to mediate repression of marker genes in vitro and in a ligation model of vascular injury in vivo. ${ }^{14,15}$

Evidence from other work supports an important role for SMCs in the pathophysiology of aortic aneurysms. Longo

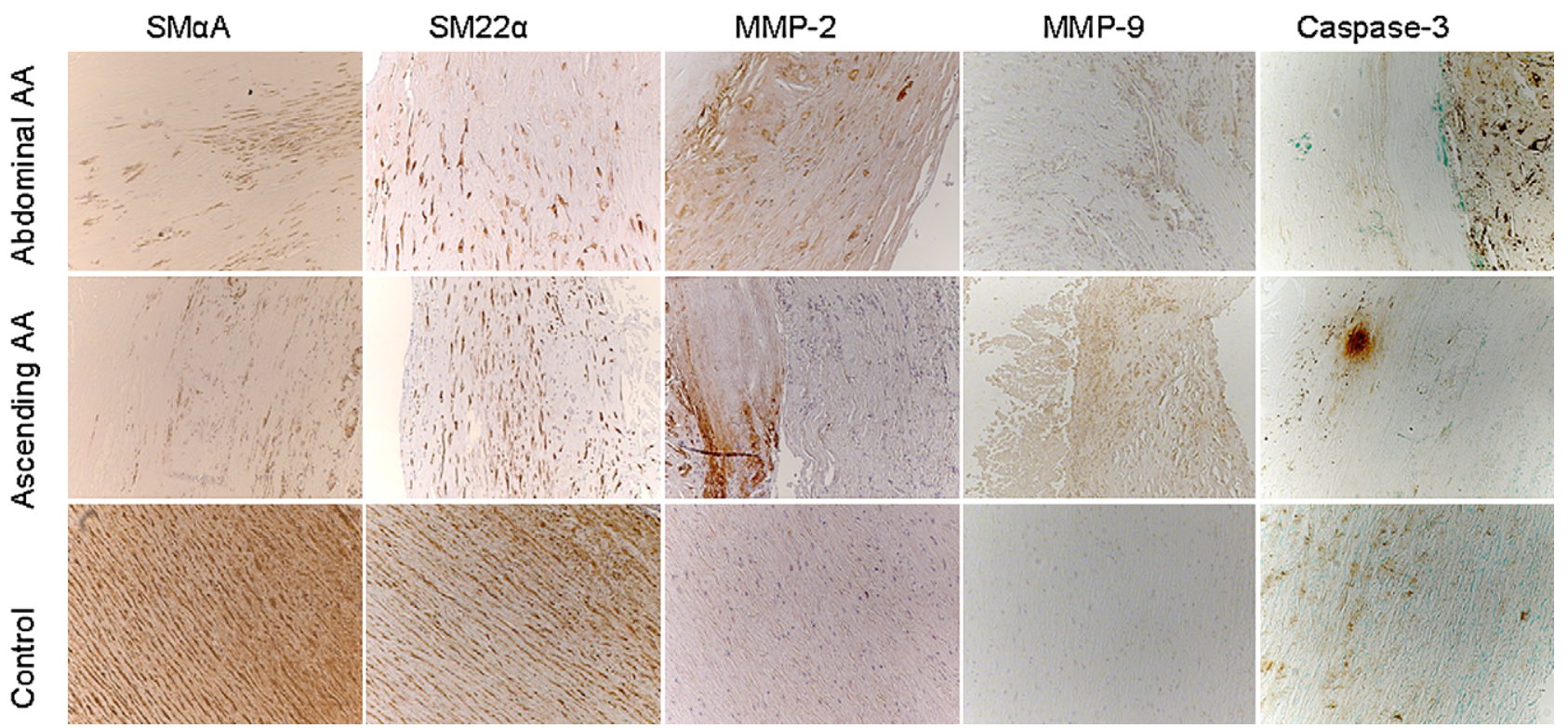

FIGURE 6. Immunohistochemistry in human ascending and abdominal aortic aneurysms (AA) and control ascending aortas. SM $\alpha$-actin and SM22 $\alpha$ are downregulated in aortic aneurysms compared with that seen in control aortas. Expression of matrix metalloproteinase $(M M P) 2$ and 9 is increased in aneurysmal tissue. Apoptosis (caspase-3) is evident in ascending and abdominal aortic aneurysms and, to a lesser extent, in control aortas. All images were obtained at $10 \times$ magnification. 
and colleagues ${ }^{16}$ suggested a critical role for SMCs in experimental aneurysm formation. MMP-2 knockout mice are resistant to experimental aortic aneurysms using a model of abluminal calcium chloride application. Reconstitution of these mice with wild-type bone marrow did not alter this resistance, suggesting that MMP-2 derived from outside the bone marrow is critical. ${ }^{17}$ It is well known that MMP-2 is expressed in high levels by SMCs in vitro in response to a number of inflammatory cytokines. There is also evidence suggesting that mutations within SMC genes contribute to at least some human familial aneurysm syndromes. For example, missense mutations in ACTA2, an isoform of SM $\alpha$ actin, result in abnormal SMC contractile function and are believed to cause approximately $14 \%$ of inherited ascending thoracic aneurysms. ${ }^{18}$ Moreover, mutations in the SMC specific myosin heavy chain gene (MYH11) are associated with ascending aortic aneurysms. ${ }^{19}$ Collectively, these findings suggest that alterations in SMCs, including production of MMPs and contractile function, likely contribute to the development of aneurysms.

There has been recent excitement regarding the role of transforming growth factor (TGF) $\beta$ in the pathogenesis of aortic aneurysms. ${ }^{17}$ However, the exact mechanisms by which TGF- $\beta$ signaling relates to abdominal aortic aneurysm formation are unclear. Inhibition of TGF- $\beta$ with antibody prevents aortic root aneurysms in the fibrillin-1 mutant (Marfan) mouse. ${ }^{20}$ Conversely, adenoviral overexpression of TGF- $\beta$ results in stabilization of abdominal aortic aneurysms in rats. ${ }^{21}$ At the leading edge of human abdominal aortic aneurysms, apoptotic SMCs colocalize with high levels of TGF- $\beta$, suggesting that TGF- $\beta$ might mediate apoptosis in aneurysms. ${ }^{22}$ During vascular development, however, TGF- $\beta$ is known to promote SMC differentiation and production of SMC marker genes in vivo and in vitro. ${ }^{23}$ In addition, mice deficient in TGF- $\beta 1$ die in utero in large part because of the failure of mesenchymal precursor cells to differentiate into vascular SMCs. ${ }^{24}$ Despite these paradoxic findings of the role of TGF- $\beta$, there appears to be an important link between TGF- $\beta$ signaling and vascular SMCs during aneurysm formation.

There are several limitations to this study. First, our findings of SMC phenotypic modulation before experimental aneurysms are correlative and not causative. To prove a causative role would require the ability to prevent or alter SMC phenotype before or after elastase perfusion.

Second, although we have experimental models that can correlate with small aneurysms, the limited access to small aneurysms (not requiring surgical treatment) in human subjects limits our ability to assess whether phenotypic modulation of SMCs occurs early in the course of human aortic aneurysms.

The elastase perfusion model has certain shortcomings that should be noted. The model does cause an initial dilation after perfusion of $30 \%$ to $50 \%$ that is well described after either elastase or saline perfusion. ${ }^{4-6}$ In addition, the elastase model is admittedly rapid, with the development of an aneurysm by 14 days. Nevertheless, this model has been extensively studied and mimics human aneurysms with the hallmark pathologic features, including matrix degradation, increased cytokine production, and the presence of a chronic inflammatory infiltrate. ${ }^{4-6}$ In addition, these SMC marker genes are known to be expressed in a variety of other cell types, including fibroblasts and myofibroblasts, in the setting of wound repair. ${ }^{25}$ As such, we focused on the medial wall of the aorta, where SMCs are the predominant cell type. We infer that MMP-2 is being produced by SMCs, among other cells, in the medial wall. MMP-2 is a constitutive enzyme produced by multiple cell types. Because there is no marker for phenotypically modulated SMCs, there is no way to confirm that modulated SMCs are the source of MMP-2 after elastase perfusion without lineage-tracing studies.

We used an abdominal aortic aneurysm model, whereas our analysis in human subjects was primarily of ascending aortic aneurysms (because of our ability to obtain ascending aortic control tissue). Importantly, the embryologic origin and risk factors for ascending and abdominal aortic aneurysms are different. As such, the pathophysiology of ascending and abdominal aneurysms might be different as well. We demonstrated a similar pattern of decreased SMC marker genes and upregulation of MMPs by means of immunohistochemistry in abdominal aortic aneurysms. However, we do not have control tissue from the abdominal aorta, making comparison somewhat indirect.

Experimental murine and human aneurysms demonstrate SMC phenotypic modulation characterized by reduced expression of SM marker genes and upregulation of MMPs. These events in experimental models occur early before discernable differences in aneurysm formation. Because SMCs can synthesize extracellular matrix proteins, they might be an ideal target for therapy. The ability to alter SMC differentiation has been marred by the fact that many of the regulators of SMC maturation, including TGF- $\beta 1$, TGF- $\beta$ receptor II, and SMAD5, result in embryonic lethality because of defects in vascular maturation, SMC differentiation, or both., ${ }^{2,3}$ Future directions aimed at understanding and altering the mechanisms that control phenotypic switching in aortic aneurysms will use the ability to conditionally knock out the regulators of SMC phenotype. Targeting SMCs to a reparative phenotype might provide a novel therapy in the treatment of aortic aneurysms.

\section{References}

1. Ailawadi G, Eliason JL, Upchurch GR Jr. Current concepts in the pathogenesis of abdominal aortic aneurysm. J Vasc Surg. 2003;38:584-8.

2. Owens GK, Kumar MS, Wamhoff BR. Molecular regulation of vascular smooth muscle cell differentiation in development and disease. Physiol Rev. 2004;84: 767-801. 
3. Kawai-Kowase K, Owens GK. Multiple repressor pathways contribute to phenotypic switching of vascular smooth muscle cells. Am J Physiol Cell Physiol. 2007; 292:C59-69.

4. Anidjar S, Salzmann JL, Gentric D, Lagneau P, Camilleri JP, Michel JB. Elastaseinduced experimental aneurysms in rats. Circulation. 1990;82:973-81.

5. Ailawadi G, Eliason JL, Roelofs KJ, et al. Gender differences in experimental aortic aneurysm formation. Arterioscler Thromb Vasc Biol. 2004;24:2116-22.

6. Eliason JL, Hannawa KK, Ailawadi G, et al. Neutrophil depletion inhibits experimental abdominal aortic aneurysm formation. Circulation. 2005;112: 232-40.

7. Layne MD, Yet SF, Maemura K, et al. Characterization of the mouse aortic carboxypeptidase-like protein promoter reveals activity in differentiated and dedifferentiated vascular smooth muscle cells. Circ Res. 2002;90:728-36.

8. Aikawa M, Sakomura Y, Ueda M, et al. Redifferentiation of smooth muscle cells after coronary angioplasty determined via myosin heavy chain expression. $\mathrm{Circu}$ lation. 1997;96:82-90.

9. Mack CP, Owens GK. Regulation of smooth muscle alpha-actin expression in vivo is dependent on CArG elements within the $5^{\prime}$ and first intron promoter regions. Circ Res. 1999;84:852-61.

10. Manabe I, Owens GK. CArG elements control smooth muscle subtype-specific expression of smooth muscle myosin in vivo. J Clin Invest. 2001;107:823-34.

11. Miano JM, Carlson MJ, Spencer JA, Misra RP. Serum response factordependent regulation of the smooth muscle calponin gene. J Biol Chem. 2000; 275:9814-22.

12. Miano JM, Long X, Fujiwara K. Serum response factor: master regulator of the actin cytoskeleton and contractile apparatus. Am J Physiol Cell Physiol. 2007; 292:C70-81.

13. Wamhoff BR, Hoofnagle MH, Burns A, Sinha S, McDonald OG, Owens GK. A $\mathrm{G} / \mathrm{C}$ element mediates repression of the SM22 alpha promoter within phenotypically modulated smooth muscle cells in experimental atherosclerosis. Circ Res. 2004;95:981-8.

14. Liu Y, Sinha S, McDonald OG, Shang Y, Hoofnagle MH, Owens GK. Kruppellike factor 4 abrogates myocardin-induced activation of smooth muscle gene expression. J Biol Chem. 2005;280:9719-27.

15. Yoshida T, Kaestner KH, Owens GK. Conditional deletion of Krüppel-like factor 4 delays downregulation of smooth muscle cell differentiation markers but accelerates neointimal formation following vascular injury. Circ Res. 2008;102: 1548-57.

16. Longo GM, Xiong W, Greiner TC, Zhao Y, Fiotti N, Baxter BT. Matrix metalloproteinases 2 and 9 work in concert to produce aortic aneurysms. $J$ Clin Invest. 2002;110:625-32.

17. Jones JA, Spinale FG, Ikonomidis JS. Transforming growth factor-beta signaling in thoracic aortic aneurysm development: a paradox in pathogenesis. J Vasc Res. 2009;46:119-37.

18. Guo DC, Pannu H, Tran-Fadulu V, et al. Mutations in smooth muscle alpha-actin (ACTA2) lead to thoracic aortic aneurysms and dissections. Nat Genet. 2007;9: 1488-93.

19. Pannu H, Tran-Fadulu V, Papke CL, et al. MYH11 mutations result in a distinct vascular pathology driven by insulin-like growth factor 1 and angiotensin II. Hum Mol Genet. 2007;16:2453-62.

20. Habashi JP, Judge DP, Holm TM, et al. Losartan, an AT1 antagonist, prevents aortic aneurysm in a mouse model of Marfan syndrome. Science. 2006;312: 117-21.

21. Dai J, Losy F, Guinault AM, et al. Overexpression of transforming growth factor betal stabilizes already-formed aortic aneurysms: a first approach to induction of functional healing by endovascular gene therapy. Circulation. 2005;112: 1008-15.

22. Fukui D, Miyagawa S, Soeda J, Tanaka K, Urayama H, Kawasaki S. Overexpression of transforming growth factor betal in smooth muscle cells of human abdominal aortic aneurysm. Eur J Vasc Endovasc Surg. 2003;25:540-5.

23. Hautmann MB, Adam PJ, Owens GK. Similarities and differences in smooth muscle alpha-actin induction by TGF-beta in smooth muscle versus non-smooth muscle cells. Arterioscler Thromb Vasc Biol. 1999;19:2049-58.

24. Martin JS, Dickson MC, Cousins FM, Kulkarni AB, Karlsson S, Akhurst RJ. Analysis of homozygous TGF-beta 1 null mouse embryos demonstrates defects in yolk sac vasculogenesis and hematopoiesis. Ann N Y Acad Sci. 1995; 752:300-8.

25. Sartore S, Chiavegato A, Faggin E, et al. Contribution of adventitial fibroblasts to neointima formation and vascular remodeling: from innocent bystander to active participant. Circ Res. 2001;89:1111-21.

\section{Discussion}

Dr John S. Ikonomidis (Charleston, SC). I have no relationships to disclose.

Gorav, this was a very interesting presentation. I have several questions for you.

First, as you mentioned, it is well described that SMCs undergo apoptosis during aneurysm development. How do you account for the negative results seen with your animal model as opposed to the results you saw in the human specimens?

Dr Ailawadi. For the reasons you note, I thought it was important to show our caspase-3 data. We have also done Ki-67 staining to assess proliferation. In our best assessment, the SMC count in our model appears to be similar, suggesting that the SMCs are present but are not expressing these marker genes. In our human samples, which are advanced lesions, there is loss of SMCs.

Dr Ikonomidis. In that regard, is it possible that the changes that you are seeing could be explained by a regional heterogeneity phenomenon? In this study you are comparing human ascending aortas with murine abnormal aortas. Is it possible that there is a body of evidence that suggests that regional heterogeneity exists within the aorta, and could that contribute to these results?

Dr Ailawadi. It is certainly possible. We have investigated the concept of regional heterogeneity in the past, and there do appear to be differences along the aorta. We did analyze abdominal aortic aneurysms, and they follow the same pattern, with downregulation of SM marker genes in these advanced human lesions that are being resected as the human ascending aorta. However, we do not have good control tissue for the abdominal aorta. Normal abdominal aortas in human subjects are difficult to access, and that is why we chose to use ascending aortas from human subjects as our correlate. We believe that many of the effector pathways involved in ascending and abdominal aortas are quite similar.

Dr Ikonomidis. Next, MMP-2 can be produced by multiple cell types. MMP-9 has been shown in this particular model to be associated with or produced by the inflammatory infiltrate. You have shown data that suggest that there is a phenotypic modulation, and you have also shown data that there is upregulation in MMP-2 and MMP-9 expression. Do you have data that specifically colocalizes production of MMP-2 and MMP-9 to a unique cell with a specific phenotype?

Dr Ailawadi. That is the real challenge when we are trying to correlate modulated SMCs. The way we analyze or identify SMCs is by their marker genes, and because they lose those marker genes, it becomes very difficult to assess that this is truly an SMC.

To answer your excellent question requires the use of unique transgenic mice. These studies are currently underway in our laboratory with genetically altered mice with SMCs that are altered to express Lac Z, even if they lose their ability to express SM22. Our preliminary studies demonstrate that SMCs still express MMP-2.

Dr Ikonomidis. The last question I have is that you are making assertions about a phenotypic change in this study based on changes in stains for an SMC actin and SM22. Do you have any data on any more direct surrogates of phenotypic change, for example, KLF-4?

Dr Ailawadi. We are doing several ongoing studies along these lines. KLF-4 is involved in the pathways of SMC modulation.

Dr Ikonomidis. Again, congratulations on this stimulating work. 